\title{
Synthesis, Characterization and Anticonvulsant Activity of Novel Fused 1,2,4-Triazolo-1,3,4-Thiadiazoles
}

\section{MOHAMMAD SARAFROZ ${ }^{1 *}$, YASMIN KHATOON ${ }^{2}$, NIYAZ AHMAD ${ }^{3}$, MOHAMMAD AMIR $^{4}$, SALAHUDDIN ${ }^{5}$ and FAHEEM HYDER POTTOO ${ }^{6}$}

\author{
1Department of Pharmaceutical Chemistry, College of Clinical Pharmacy, Imam Abdulrahman Bin Faisal \\ University, P.O. Box 1982, City Dammam, Saudi Arabia. \\ ${ }^{2}$ School of Pharmacy, Department of Pharmaceutical Chemistry, Sharda University, Knowledge Park III, \\ Greater Noida, Uttar Pradesh, India. \\ ${ }^{3}$ Department of Pharmaceutics, College of Clinical Pharmacy, Imam Abdulrahman Bin Faisal University, \\ P.O. Box 1982, City Dammam, Saudi Arabia. \\ ${ }^{4}$ Department of Pharmacognosy and Phytochemistry, College of Clinical Pharmacy, Imam Abdulrahman \\ Bin Faisal University, P.O. Box 1982, City Dammam, Saudi Arabia. \\ ${ }^{5}$ Department of Pharmaceutical Chemistry, Noida institute of Engineering and Technology (Pharmacy Institute), \\ Greater Noida, Uttar Pradesh, India. \\ ${ }^{6}$ Department of Pharmacology, College of Clinical Pharmacy, Imam Abdulrahman Bin Faisal University, \\ P.O. Box 1982, City Dammam, Saudi Arabia. \\ ${ }^{\star}$ Corresponding author E-mail: drsarafroz @gmail.com \\ http://dx.doi.org/10.13005/ojc/350107
}

(Received: October 01, 2018; Accepted: February 01, 2019)

\section{ABSTRACT}

A novel category of 1,2,4-triazolo-1,3,4-thiadiazoles were ready by the utilization of 3-amino-4hydroxybenzoate as the beginning material. Spectral information results were used for the establishing of prepared compounds. Compounds were screened anticonvulsant activity for obtaining better results by MES test and ScPTZ methods. The rotarod method was used for neurotoxicity analysis. Majority of the compounds displayed distinguished anticonvulsant impact practically identical to standard drugs (phenytoin and carbamazepine) with slight neurotoxicity.

Keywords: Substituted triazolo-thiadiazole, Anticonvulsant, Neurotoxicity and lipophilicity.

\section{INTRODUCTION}

Convulsion is a condition of brain described via the irregular and unreliable episode of seizures. It is a collective neurological disorder, affecting $0.5-1 \%$ of the people globally according to epidemiological studies $^{1-3}$. Each year around 0.25 million new cases are added to this figures ${ }^{4-5}$. More than twenty antiepileptic drugs of different classes (like carbamazepine, phenytoin, valproate,

This is an Open Access article licensed under a Creative Commons license: Attribution 4.0 International (CC- BY). Published by Oriental Scientific Publishing Company @ 2018

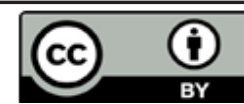


phenobarbital, vigabatrin, lamotrigine, tiagabine, topiramate, levetiracetam and gabapentin) are available for the treatment of seizures ${ }^{6}$. Despite introduction of these novel AEDs, the management of epilepsy quiet insufficient and part of the patients suffers from several complications like neurotoxicity; symptoms of depression, CNS associated disorders, gingival hypertrophy, liver toxicity and megaloblastic anemias $\mathrm{s}^{7-8}$. In adequacy and toxicities are the restrictions of the present drugs ${ }^{9-10}$ because of these restrictions there is a critical need to create new antiepileptic agents with increase seizure control, increased acceptability, improved protection and pharmacokinetic properties with neuroprotective actions ${ }^{11}$.

In present times, an interest of fused triazolothiadiazole derivatives has received impressive consideration because of their biological significance. Compounds having triazole or thiadiazole as a core moiety are represented for wide range of pharmacological properties for example anticancer ${ }^{12}$, anti-inflammatory ${ }^{13}$, analgesic ${ }^{14}$, antimicrobial ${ }^{15}$, antibacterial ${ }^{16}$, antifungal ${ }^{17}$, insecticidal ${ }^{18}$, herbicidal ${ }^{19}$ and CNS stimulating ${ }^{20}$. Fused triazolo-thiadiazole derivatives were found to diverse biological activities for example, anti-inflammatory ${ }^{21-23}$, antimicrobial ${ }^{24-25}$ and anticancer ${ }^{26-27}$. Triazolothiadiazole, a heterocyclic compound of diverse biological activities was observed to be one of the novel classes of anticonvulsants as discovered by literature study ${ }^{28}$. Recently, the area of antiepileptic drug development (ADD) has turned out to be very unique, bearing numerous promising examination openings, and is proceeding with interest for new chemical entities because it is difficult to manage each sort of seizure with existing medications.

Literature review reveals that substituted triazolo-thiadiazoles were not given careful thought for antiepileptic drug actions. Therefore, it was thought to plan and synthesize a combination of 1,2,4-triazolo-1,3,4-thiadiazoles as a fundamental core joined with substituted benzoxazole moiety within a single frame. Such combination is would have to make compounds with lipophilic character having promising anticonvulsant actions.

\section{MATERIALS AND METHODS}

\section{Chemistry}

The liquid paraffin bath was used for determination of melting points $\left({ }^{\circ} \mathrm{C}\right)$ and was uncorrected. The laboratory solvents were purchased from Spectrochem, C.D.H and S.D. Fine chem. Ltd. The different solvent systems were used for detection of completion of chemical reactions through TLC method. The iodine and UV light chamber were utilized for imagining of spots. The ${ }^{1} \mathrm{H}-\mathrm{NMR}$ spectrums were noted on Bruker $300 \mathrm{MHz}$ and 400 Ultra ShieldTM spectrometer in $\mathrm{DMSO} / \mathrm{d}_{6}$ and values are expressed in ppm in respect to tetramethylsilane. The FT-IR spectrophotometer (BIO-RAD FTS) was used for IR bands utilizing $\mathrm{KBr}$ pellets. UPLC-MS/ MS spectrometer (WATERS, Mass Lynx version 4.1) was used for mass spectrum and elemental analysis was performed using Perkin-Elmer 2400 and found within $\pm 0.4 \%$ of theoretical values.

\section{Synthesis of benzoxazole hydrazide (2)}

These compounds were obtained from methyl 3-amino-4-hydroxybenzoate by the outline described in the literature ${ }^{30-31}$.

\section{Synthesis of compound (3)}

Equimolar quantities of acid hydrazide (2) and carbon disulfide were stirred for $12 \mathrm{~h}$ in presence of $50 \mathrm{ml}$ alcoholic potassium hydroxide solution at room temperature. Finally the reaction mixtures were cooled and poured onto ice. The potassium dithiocarbazinate, which isolated out, was separated, washed with ether and dried ${ }^{28,30}$.

\section{Synthesis of compound (4)}

In this reaction compound (3) and hydrazine hydrate (1:2) was refluxed for 10-15 $\mathrm{h}$ in water $(50 \mathrm{~mL})$. The reaction materials were cooled and acidify with hydrochloric acid. The resultant solid precipitate of 1,2,4-triazole separated out, splashed with ice water, desiccated and recrystallized from ethanol to give 4 in good yield ${ }^{28,31}$.

\section{General protocol for the synthesis of $1,2,4-$ triazolo-1,3,4-thiadiazoles (5a-l)}

The compound (4) and different substituted/ unsubstituted aryl acids (1:1) in $10 \mathrm{ml}$ of phosphoryl chloride was condensed for 5-6 hours. successively the contents were stirred on ice cold water for 4-5 hours. The content was permitted to stand overnight, the precipitate separated, neutralized with aqueous alkali, dehydrated and purified from ethanol ${ }^{28,32}$.

\section{Compound (5a)}

Yield $80 \%$; m.p. $145-147^{\circ} \mathrm{C}$; IR $\left(\mathrm{KBr}, \mathrm{cm}^{-1}\right)$ : 
3033 (CH str), 1611 (C=N, cyclic), 1480 (C-N, cyclic), 1227 (N-N=C, cyclic), 722 (C-Cl), 711 (C-S-C, cyclic); ${ }^{1} \mathrm{H}-\mathrm{NMR}$ (DMSO-d $\left.{ }_{6}\right) \delta$ (ppm): 7.00-8.11 (m, 11HAromatic), $3.86\left(\mathrm{~s}, 3 \mathrm{H}, \mathrm{OCH}_{3}\right)$. Elemental Analysis $\left(\mathrm{C}_{23} \mathrm{H}_{14} \mathrm{CIN}_{5} \mathrm{O}_{2} \mathrm{~S}\right)$ : Calcd. C, 59.83; H, 3.01; N, 14.98; Found: C, 60.07; H, 3.07; N, 15.23.

\section{Compound (5b)}

Yield 81\%; m.p. 165-167 C; IR ( $\left.\mathrm{KBr}, \mathrm{cm}^{-1}\right)$ : 3032 (CH str), 1641 (C=N, cyclic), 1492 (C-N, cyclic), 1218 (N-N=C, cyclic), 741 (C-Cl), 701 (C-S-C); ${ }^{1} \mathrm{H}-\mathrm{NMR}\left(\mathrm{DMSO}-\mathrm{d}_{6}\right.$ ) $\delta$ (ppm): 7.34-8.19 (complex, m, 16H-Aromatic). Mass (m/z): $505(\mathrm{M}+1)$; Elemental Analysis $\left(\mathrm{C}_{28} \mathrm{H}_{16} \mathrm{CIN}_{5} \mathrm{OS}\right)$ : Calcd. C, 66.65; H, 3.46:, N, 14.12; Found: C, 66.47; H, 3.19; N, 13.84.

\section{Compound (5c)}

Yield $56 \%$; m.p. $155-157^{\circ} \mathrm{C}$; IR $\left(\mathrm{KBr}, \mathrm{cm}^{-1}\right)$ : 3023 (CH str), 1655 (C=N, cyclic), 1475 (C-N, cyclic), 1214 (N-N=C, cyclic), 766 (C-Cl), 698 (C-S-C, cyclic); ${ }^{1} \mathrm{H}-\mathrm{NMR}\left(\mathrm{DMSO}-\mathrm{d}_{6}\right.$ ) $\delta$ (ppm): 7.15-7.99 (complex, m, 17H-Aromatic), 5.11 (s, $1 \mathrm{H} \mathrm{CH}$, diphenyl). Elemental Analysis $\left(\mathrm{C}_{28} \mathrm{H}_{16} \mathrm{CIN}_{5} \mathrm{OS}\right)$ : Calcd. C, 66.65; $\mathrm{H}, 3.46$; N, 14.12; Found: C, 66.47; H, 3.19; N, 13.84.

\section{Compound (5d)}

Yield $70 \%$; m.p. $120-122^{\circ} \mathrm{C}$; IR $\left(\mathrm{KBr}, \mathrm{cm}^{-1}\right)$ : 3036 (CH str), 1591 (C=N, cyclic), 1478 (C-N, cyclic), 1239 (N-N=C, cyclic), 723 (C-Cl), 717 (C-S-C, cyclic); ${ }^{1} \mathrm{H}-\mathrm{NMR}$ (DMSO-d ${ }_{6}$ ) $\delta$ (ppm): 6.90-7.79 (m, 12H-Aromatic), 5.06 (s, 2H, $\mathrm{OCH}_{2}$ ); Mass (m/z): $459(\mathrm{M}+1)$; Elemental Analysis $\left(\mathrm{C}_{23} \mathrm{H}_{14} \mathrm{ClN}_{5} \mathrm{O}_{2} \mathrm{~S}\right)$ : Calcd. C, 59.58; H, 3.43; N, 14.91; Found: C, 60.07; $\mathrm{H}, 3.07 ; \mathrm{N}, 15.23$.

\section{Compound (5e)}

Yield $72 \%$; m.p. $195-197^{\circ} \mathrm{C}$; IR $\left(\mathrm{KBr}, \mathrm{cm}^{-1}\right)$ : 3065 (CH str), 1733 (carbonyl), 1611 (C=N, cyclic), 1489 (C-N, cyclic), 1223 (N-N=C, cyclic), 720 (C-Cl), 711 (C-S-C, cyclic); ${ }^{1} \mathrm{H}-\mathrm{NMR}$ (DMSO-d ${ }_{6}$ ) $\delta$ (ppm): 10.11 (s, $1 \mathrm{H}, \mathrm{CHO}), 7.29-8.08$ (m, 11HAromatic), 5.08 (s, $2 \mathrm{H}, \mathrm{OCH}_{2}$ ); Elemental Analysis $\left(\mathrm{C}_{24} \mathrm{H}_{14} \mathrm{ClN}_{5} \mathrm{O}_{3} \mathrm{~S}\right)$ : Calcd. C, 59.24; H, 3.08; N, 13.99; Found: C, 59.08; H, 2.89; N, 14.35.

\section{Compound (5f)}

Yield 75\%; m.p. $160-162^{\circ} \mathrm{C}$; IR $\left(\mathrm{KBr}, \mathrm{cm}^{-1}\right)$ : 3033 (CH str), 1738 (carbonyl), 1623 (C=N, cyclic), 1490 (C-N, cyclic), 1223 (N-N=C, cyclic), 724 (C-Cl), 692 (C-S-C, cyclic); ${ }^{1} \mathrm{H}-\mathrm{NMR}$ (DMSO-d 6 ) $\delta$ (ppm): 10.27 (s, 1H, CHO), 7.36-8.01 (m, 11H-Aromatic);
Mass (m/z): $457(\mathrm{M}+1)$; Elemental Analysis $\left(\mathrm{C}_{23} \mathrm{H}_{12} \mathrm{CIN}_{5} \mathrm{O}_{2} \mathrm{~S}\right)$ : Calcd. C, 60.41; $\mathrm{H}, 2.23$; N, 15.11; Found: C, 60.33; H, 2.64; N, 15.29.

\section{Compound (5g)}

Yield $80 \%$; m.p. $140-142^{\circ} \mathrm{C}$; IR $\left(\mathrm{KBr}, \mathrm{cm}^{-1}\right)$ : 3020 (CH str), 1611 (C=N, cyclic), 1498 (C-N, cyclic), 1256 (N-N=C, cyclic), 757 (C-Cl), 698 (C-S-C, cyclic); ${ }^{1} \mathrm{H}-\mathrm{NMR}\left(\mathrm{DMSO}-\mathrm{d}_{6}\right.$ ) $\delta$ (ppm): 7.39-8.17 (m, complex, $11 \mathrm{H}$-Aromatic); Elemental Analysis $\left(\mathrm{C}_{21} \mathrm{H}_{11} \mathrm{CIN}_{6} \mathrm{OS}\right)$ : Calcd. C, 58.13; H, 3.01; N, 19.11; Found: C, 58.54; $\mathrm{H}, 2.57 ; \mathrm{N}, 19.50$.

\section{Compound (5h)}

Yield 63\%; m.p. $150-152^{\circ} \mathrm{C}$; IR $\left(\mathrm{KBr}, \mathrm{cm}^{-1}\right)$ : 3037 (CH str), 1601 (C=N, cyclic), 1484 (C-N, cyclic), 1244 (N-N=C, cyclic), 714 (C-Cl), 700 (C-S-C, cyclic); ${ }^{1} \mathrm{H}-\mathrm{NMR}\left(\mathrm{DMSO}-\mathrm{d}_{6}\right) \delta$ (ppm): 7.10-7.98 (m, 12HAromatic), $3.48\left(2 \mathrm{H}, \mathrm{m}, \mathrm{CH}_{2}\right)$; Elemental Analysis (C23H14CIN5OS): Calcd. C, 62.10; H, 3.15; N, 15.80; Found: C, 62.23; H, 3.18; N, 15.78.

\section{Compound (5i)}

Yield 60\%; m.p. $180-182^{\circ} \mathrm{C}$; IR $\left(\mathrm{KBr}, \mathrm{cm}^{-1}\right)$ : 3576 (OH str), 2998 (CH str), 1608 (C=N, cyclic), 1490 (C-N, cyclic), 1235 (N-N=C), 734 (C-Cl), 701 (C-S-C, cyclic); ${ }^{1} \mathrm{H}-\mathrm{NMR}$ (DMSO-d ${ }_{6}$ ) $\delta$ (ppm): 1078 (s, 3H, $3 \times \mathrm{OH}), 6.86-8.98$ (m, 9H-Aromatic); Mass (m/z): $477(\mathrm{M}+1)$; Elemental Analysis $\left(\mathrm{C}_{22} \mathrm{H}_{12} \mathrm{CIN}_{5} \mathrm{O}_{4} \mathrm{~S}\right)$ : Calcd. C, 55.01; $\mathrm{H}, 2.44 ; \mathrm{N}, 14.83$; Found: C, 55.29; H, 2.53; N, 14.66.

\section{Compound (5j)}

Yield $67 \%$; m.p. $175-177^{\circ} \mathrm{C}$; IR $\left(\mathrm{KBr}, \mathrm{cm}^{-1}\right)$ : 2996 (CH str), 1604 (C=N, cyclic), 1489 (C-N, cyclic), 1220 (N-N=C), 719 (C-Cl), 697 (C-S-C, cyclic); ${ }^{1} \mathrm{H}-\mathrm{NMR}\left(\mathrm{DMSO}-\mathrm{d}_{6}\right.$ ) $\delta$ (ppm): 7.30-8.11 (m, complex, 14H-Aromatic), 4.38 (s, 2H, $\mathrm{CH}_{2}$ ); Mass (m/z): 493 $(\mathrm{M}+1)$; Elemental Analysis $\left(\mathrm{C}_{27} \mathrm{H}_{16} \mathrm{CIN}_{5} \mathrm{OS}\right)$ : Calcd. C, 65.71; H, 3.12; N, 14.30; Found: C, 65.65; H, $3.26 ; \mathrm{N}, 14.10$.

\section{Compound (5k)}

Yield 51\%; m.p. $165-167^{\circ} \mathrm{C}$; IR $\left(\mathrm{KBr}, \mathrm{cm}^{-1}\right)$ : 3564 (OH str), 3004 ( $\mathrm{CH}$ str), 1603 ( $\mathrm{C}=\mathrm{N}$, cyclic), 1474 (C-N, cyclic), 1214 (N-N=C), 733 (C-Cl), 691 (C-S-C, cyclic); ${ }^{1} \mathrm{H}-\mathrm{NMR}$ (DMSO-d 6 ) $\delta$ (ppm): 10.11 (s, 1H, OH), 6.92-7.83 (m, 11H-Aromatic); Elemental Analysis $\left(\mathrm{C}_{22} \mathrm{H}_{12} \mathrm{CIN}_{5} \mathrm{O}_{2} \mathrm{~S}\right)$ : Calcd. C, 58.91; $\mathrm{H}, 3.01$; N, 15.83; Found: C, 59.26; H, 2.71; N, 15.71. 


\section{Compound (5l)}

Yield 62\%; m.p. $155-157^{\circ} \mathrm{C}$; IR $\left(\mathrm{KBr}, \mathrm{cm}^{-1}\right)$ : 3033 ( $\mathrm{CH}$ str), 1730 (O-C=O), 1583 ( $\mathrm{C}=\mathrm{N}$, cyclic), 1463 (C-N, cyclic), 1260 (N-N=C), 690 (C-S-C, cyclic); ${ }^{1} \mathrm{H}-\mathrm{NMR}$ (DMSO-d $\mathrm{d}_{6}$ ) $\delta$ (ppm): 7.06-8.07 (m, $11 \mathrm{H}$-Aromatic), $2.48\left(\mathrm{~s}, 3 \mathrm{H}, \mathrm{CH}_{3}\right)$; Elemental Analysis (C24H14CIN5O3S): Calcd. C, 58.88; H, 3.01; N, 13.98; Found: C, 59.08; H, 2.89; N, 14.35 .

\section{Pharmacological screening}

The anticonvulsant screening of the final compounds (5a-l) were tested on Swiss albino mice (20-25 g), permitted by the Institutional Animal Ethics committee, R.V. Northland Institute, Dadri, Greater Noida, Uttar Pradesh-India, under the proposal number RVNI//AEC/2017/05. The method was followed conferring to the Antiepileptic Drug Development Program ${ }^{33-34}$. All the newly synthesized compounds (5a-l) were suspended in polyethylene glycol (PEG400) and administered intraperitoneally.

\section{The Maximal electroshock Seizure test}

This method uses a current intensity of $50 \mathrm{~mA}, 60 \mathrm{~Hz}$, connected by means of corneal terminals for $0.2 \mathrm{~s}$ for induction of seizures. The maximal seizure consistently involves a little time of tonic extension of the hind limbs and a last clonic scene. Absence of even a threshold seizure was noted as an estimation of antiseizure action ${ }^{34}$.

\section{The scPTZ seizure test}

The assessment method was taken after with reference to the known protocol ${ }^{35}$. This procedure uses $85 \mathrm{mg} / \mathrm{kg}$ of scPTZ that causes attacks in $\pm 97 \%$ of the mice. The experimental candidates were administered half an hour before the ScPTZ; the defend was identified in relations of absence of a scene of clonic spasm for $5 \mathrm{sec}$ duration showed a candidate's capacity to nullify the impact of PTZ happening seizure edge.

\section{Minimal motor impairment (neurotoxicity) screening}

The technique was taken after with reference to the known protocol ${ }^{36}$. The mice could remain on a fast-tracking rod $(10 \mathrm{rpm}, 3.2 \mathrm{~cm}$ diameter). Those group of mice were used for motor impairment study, which could remain proceeding the spinning pole for not less than one min. Motor incapacity was appeared by the disappointment of the mice to keep up adjust on the bar for not less than 1 minutes.

\section{Lipophilicity determination}

The calculation method was taken after with reference to the known protocol ${ }^{38}$. The congener acts on CNS were associated with lipophilicity ${ }^{37}$ and it was revealed that those medications which follow up on focal sensory system, having an ideal lipophilic character $(\log P \approx 2)$. Here we endeavored to relate the antiepileptic action of the titled compounds with their figured log P standards.

\section{RESULT AND DISCUSSION}

A novel class of 1,2,4-triazolo-1,3,4thiadiazoles were prepared in appropriate yields as demonstrated in scheme 1 and the compounds were confirmed through elemental and spectral results, are described in experimental procedures.

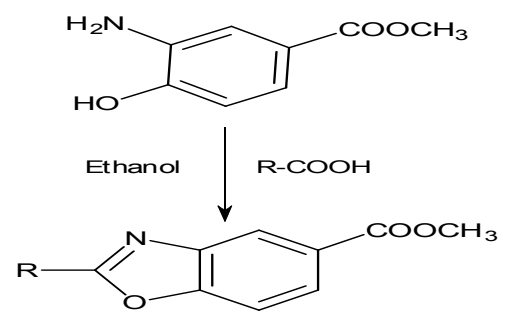

(1)

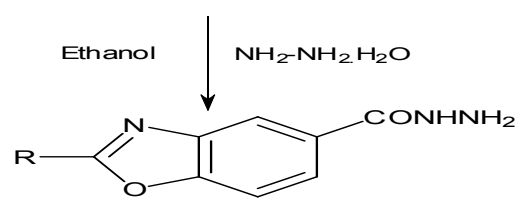

(2)

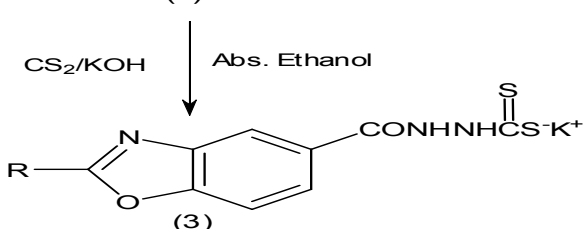

(3)

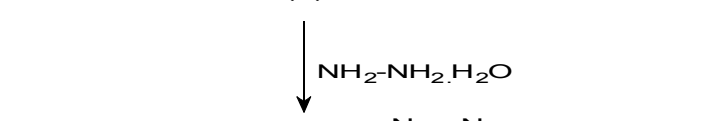<smiles>[2H]c1nc2cc(-c3nnc(S)n3N)ccc2o1</smiles>

(4)

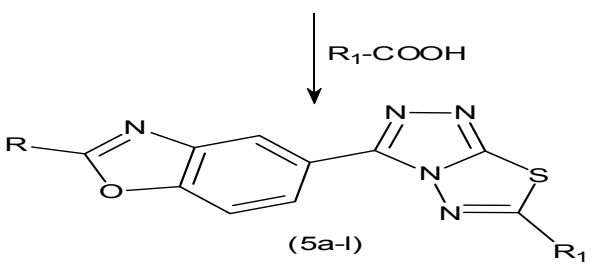

Scheme 1: Synthesis of novel triazolo-thiadiazoles 
The starting material substituted benzoate are cyclized to substituted benzoxazole carboxylate (1) on treatment with 3-chlorobenzoic acid in alcohol, which on treatment with hydrazine hydrate, afforded acid hydrazide $(2)^{29,30}$. Potassium dithiocarbazinate (3) was prepared in alcoholic $\mathrm{KOH}$ with $\mathrm{CS}_{2}^{28,29}$, which on additionally condensed with hydrazine to afforded substituted benzoxazole mercapto-traiazole (4) through direct hydrazinolysis method ${ }^{28,39}$, which was condensed with different acids in phosphoryl chloride to yield the titled compounds 1,2,4-triazolo- 1,3,4-thiadiazoles (5a-I). The structure of compound (4) was declared by ${ }^{1} \mathrm{H}-\mathrm{NMR}$ spectral results. The ${ }^{1} \mathrm{H}-\mathrm{NMR}$ spectra demonstrated that the -SH moiety appeared as singlet at $\delta$ 13.21-13.62, while the $-\mathrm{NH}_{2}$ moiety showed up as a singlet at $\delta$ 5.445.62. Although in the ${ }^{1} \mathrm{H}-\mathrm{NMR}$ spectra of triazolothiadiazoles the indications of $-\mathrm{NH}_{2}$ and $-\mathrm{SH}$ protons disappeared, affirming that triazole was converted into triazolo-thiadiazoles by reaction with different aromatic acid derivatives.

Table 1: Physical date of the titled molecules (5a-I)

\begin{tabular}{|c|c|c|c|c|c|c|}
\hline Compound & $\mathrm{R}$ & $\mathrm{R}_{1}$ & Mol. formula ${ }^{a}$ & $\mathrm{~m} \cdot \mathrm{p}^{\mathrm{b}}\left({ }^{\circ} \mathrm{C}\right)$ & $\log P c$ & $\mathrm{R}_{f}^{\mathrm{d}}$ Value \\
\hline $5 a$ & $3-\mathrm{ClC}_{6} \mathrm{H}_{4}$ & $4-\mathrm{OCH}_{3} \mathrm{C}_{6} \mathrm{H}_{4}$ & $\mathrm{C}_{23} \mathrm{H}_{14} \mathrm{CIN}_{5} \mathrm{O}_{2} \mathrm{~S}$ & $145-146$ & 1.88 & 0.80 \\
\hline $5 b$ & $3-\mathrm{ClC}_{6} \mathrm{H}_{4}^{4}$ & $4-\mathrm{C}_{6} \mathrm{H}_{5} \mathrm{C}_{6} \mathrm{H}_{4}^{4}$ & $\mathrm{C}_{28} \mathrm{H}_{16} \mathrm{CIN}_{5} \mathrm{OS}$ & $165-166$ & 1.92 & 0.81 \\
\hline $5 c$ & $3-\mathrm{ClC}_{6} \mathrm{H}_{4}$ & 2- $\mathrm{C}_{6} \mathrm{H}_{5} \mathrm{C}_{6} \mathrm{H}_{5} \mathrm{CH}$ & $\mathrm{C}_{28} \mathrm{H}_{16} \mathrm{CIN}_{5} \mathrm{OS}$ & $155-157$ & 0.98 & 0.61 \\
\hline $5 d$ & $3-\mathrm{ClC}_{6} \mathrm{H}_{4}$ & $\mathrm{C}_{6} \mathrm{H}_{5} \mathrm{OCH}_{2}$ & $\mathrm{C}_{23} \mathrm{H}_{14} \mathrm{CIN}_{5} \mathrm{O}_{2} \mathrm{~S}$ & $110-112$ & 1.90 & 0.70 \\
\hline $5 e$ & $3-\mathrm{ClC}_{6} \mathrm{H}_{4}$ & 4- $\mathrm{CHOC}_{6} \mathrm{H}_{4} \mathrm{OCH}_{2}$ & $\mathrm{C}_{24} \mathrm{H}_{14} \mathrm{CIN}_{5} \mathrm{O}_{3} \mathrm{~S}$ & 195-196 & 2.18 & 0.70 \\
\hline $5 f$ & $3-\mathrm{ClC}_{6} \mathrm{H}_{4}$ & 4- $\mathrm{CHOC}_{6} \mathrm{H}_{4}$ & $\mathrm{C}_{23} \mathrm{H}_{12} \mathrm{CIN}_{5} \mathrm{O}_{2} \mathrm{~S}$ & $160-162$ & 1.87 & 0.75 \\
\hline $5 g$ & $3-\mathrm{ClC}_{6} \mathrm{H}_{4}$ & $\mathrm{C}_{5} \mathrm{H}_{4} \mathrm{~N}$ & $\mathrm{C}_{21} \mathrm{H}_{11} \mathrm{CIN}_{6} \mathrm{OS}$ & $140-142$ & 2.11 & 0.80 \\
\hline $5 \mathrm{~h}$ & $3-\mathrm{ClC}_{6} \mathrm{H}_{4}$ & $\mathrm{C}_{6} \mathrm{H}_{5} \mathrm{CH}_{2}$ & $\mathrm{C}_{23} \mathrm{H}_{14} \mathrm{CIN}_{5} \mathrm{OS}$ & $150-151$ & 1.03 & 0.63 \\
\hline $5 i$ & $3-\mathrm{ClC}_{6} \mathrm{H}_{4}$ & $3,4,6-\mathrm{OH}-\mathrm{C}_{6} \mathrm{H}_{2}$ & $\mathrm{C}_{22} \mathrm{H}_{12} \mathrm{CIN}_{5} \mathrm{O}_{4} \mathrm{~S}$ & $180-182$ & 2.19 & 0.60 \\
\hline $5 j$ & $3-\mathrm{ClC}_{6} \mathrm{H}_{4}$ & $\mathrm{C}_{10} \mathrm{H}_{7} \mathrm{CH}_{2}$ & $\mathrm{C}_{27} \mathrm{H}_{16} \mathrm{CIN}_{5} \mathrm{OS}$ & $140-142$ & 1.09 & 0.82 \\
\hline $5 \mathrm{k}$ & $3-\mathrm{ClC}_{6} \mathrm{H}_{4}$ & $2-\mathrm{OH} \mathrm{C}{ }_{6} \mathrm{H}_{4}$ & $\mathrm{C}_{22} \mathrm{H}_{12} \mathrm{ClN}_{5} \mathrm{O}_{2} \mathrm{~S}$ & $175-176$ & 1.56 & 0.81 \\
\hline 51 & $3-\mathrm{ClC}_{6} \mathrm{H}_{4}$ & $2-\mathrm{OCOCH}_{3} \mathrm{C}_{6} \mathrm{H}_{4}$ & $\mathrm{C}_{24} \mathrm{H}_{14} \mathrm{CIN}_{5} \mathrm{O}_{3} \mathrm{~S}$ & $155-156$ & 1.89 & 0.62 \\
\hline
\end{tabular}

aSolvent of crystallization ethanol.

${ }^{\mathrm{b}} \mathrm{m}$.p of the compounds at their dissolution.

'Log $\mathrm{P}$ was calculated using absorbance data, chloroform / phosphate buffer at $28^{\circ} \mathrm{C}$.

${ }^{d}$ Solvent system - benzene :acetone (8:2, v/v), benzene:ethanol (2:0.5, v/v), toluene:ethylacetate:formic acid (5:4:1, v/v/v).

Table 2: Anticonvulsant and neurotoxicity screening of the titled molecules (5a-l)

\begin{tabular}{ccccccc}
\hline Compound & \multicolumn{3}{c}{$\begin{array}{c}\text { Intraperitoneal dose in } \\
\text { MES screensc }\end{array}$} & \multicolumn{2}{c}{ PTZ screen } & \multicolumn{2}{c}{ Neurotoxicity screena } \\
& $0.5 \mathrm{~h}$ & $4 \mathrm{~h}$ & $0.5 \mathrm{~h}$ & $4 \mathrm{~h}$ & $0.5 \mathrm{~h}$ & $4 \mathrm{~h}$ \\
\hline $5 \mathrm{a}$ & 100 & 300 & - & - & 300 & - \\
$5 \mathrm{~b}$ & 100 & - & 300 & - & - & 300 \\
$5 \mathrm{c}$ & - & - & - & - & $\times$ & $\times$ \\
$5 \mathrm{~d}$ & 100 & 300 & - & 300 & 300 & - \\
$5 \mathrm{e}$ & 30 & 300 & 300 & - & - & 300 \\
$5 \mathrm{f}$ & 100 & 300 & 300 & 300 & 300 & - \\
$5 \mathrm{~g}$ & 30 & 300 & - & 300 & - & - \\
$5 \mathrm{~h}$ & - & - & - & - & $\times$ & $\times$ \\
$5 \mathrm{i}$ & 30 & 300 & - & 300 & - & - \\
$5 \mathrm{j}$ & - & 300 & - & - & 300 & - \\
$5 \mathrm{k}$ & 300 & - & - & - & - & - \\
$5 \mathrm{l}$ & 100 & 300 & - & - & 300 & - \\
Phenytoinb & 30 & 30 & - & - & 100 & 100 \\
Carbamazepineb & 30 & 100 & 100 & 300 & 300 & 300 \\
\hline
\end{tabular}

${ }^{a}$ Doses of 30,100 and $300 \mathrm{mg} / \mathrm{kg}$ were managed to mice via i.p.route. The mice were observed 0.5 and $4 \mathrm{~h}$ after the treatment. The trace $(-)$ specifies nonappearance of activity at higher dose $(300 \mathrm{mg} / \mathrm{kg})$ and cross $(\times)$ means not screened. Propylene glycol $(0.1 \mathrm{ml}$, i.p.) was utilized as controller.

${ }^{b}$ Data of standard drugs (phenytoin and carbamazepine) were gotten referring ${ }^{40}$. 
The anticonvulsant activity of the final compounds was examined by the traditional method given by the epilepsy division of the NINDS conferring to the Antiepileptic Drug Development program ${ }^{33-35}$. Moreover, intense lethality of antiepileptic medicates in rodents is constantly showed by neurological deficits can be identified by the rotarod test ${ }^{36}$.

In the anticonvulsant activity, each compound showed action except for $5 \mathrm{c}$ and $5 \mathrm{~h}$. Compound $5 \mathrm{e}, 5 \mathrm{~g}$ and $5 \mathrm{i}$ were seen to be extremely powerful at dosage of $30 \mathrm{mg} / \mathrm{kg}$ against the MES method at $0.5 \mathrm{~h}$ time intervals characteristic for their capacity to maintain a strategic distance from seizure spread at by and large cut down dosage. Compounds viz. 5a, 5b, 5d, $5 f$ and $5 \mathrm{I}$ that indicated protection at a moderate level compared to MES model at dosage of $100 \mathrm{mg} / \mathrm{kg}$. The activity of molecules like $5 \mathrm{a}, 5 \mathrm{~d}$, $5 e, 5 f, 5 g, 5 i$ and $5 \mathrm{l}$ indicated at both time intervals. In this way, the larger part of the molecules exhibited anticonvulsant activities at $0.5 \mathrm{~h}$ interim having a quick beginning and smaller span of movement. In scPTZ examination, every compound except $5 \mathrm{a}, 5 \mathrm{c}, 5 \mathrm{~h}, 5 \mathrm{j}, 5 \mathrm{k}$ and $5 \mathrm{l}$ demonstrated movement characteristic of their capacity to counteract seizure attack. Compounds $5 \mathrm{~b}$ and $5 \mathrm{e}$ indicated $100 \%$ safety at a dosage of $300 \mathrm{mg} / \mathrm{kg}$ at $0.5 \mathrm{~h}$ and have rapid beginning however for short term of activity. A couple of compounds like $5 \mathrm{~d}, 5 \mathrm{~g}$ and $5 \mathrm{i}$ were likewise dynamic after $4.0 \mathrm{hrs}$ expanded time of activity. Just a single compound (5f) established activity at higher dose $(300 \mathrm{mg} / \mathrm{kg})$ on both time intervals. In neurotoxicity screening, rotarod test was utilized to measure the undesired impacts of the synthesized compounds, like sedation and ataxia. Compounds $5 \mathrm{~g}, 5 \mathrm{i}$ and $5 \mathrm{k}$ showed no any mortality at the higher dose $(300 \mathrm{mg} / \mathrm{kg})$. No any compounds were lethal at 0.5 and $4.0 \mathrm{hrs}$. however compounds $5 a, 5 d, 5 f, 5 j$ and $5 \mathrm{l}$ demonstrated lethality after $0.5 \mathrm{~h}$ and don't indicate harmfulness after 4.0 hours. Only two compounds ( $5 \mathrm{~b}$ and $5 \mathrm{e}$ ) exhibited delayed toxicity simply after $4.0 \mathrm{~h}$ is practically identical of standard drug carbamazepine ( $300 \mathrm{mg} / \mathrm{kg})$. Though, every molecule was less harmful than standard drug phenytoin $(100 \mathrm{mg} / \mathrm{kg})$. The compounds $5 \mathrm{a}$, $5 b, 5 d, 5 e, 5 f, 5 g, 5 i$ and 5 identified as additional hydrophobic character having strong anticonvulsant action. Compound 5j and 5k were also hydrophobic having little intensity. Only two compounds (5c and $5 \mathrm{~h})$ were a lesser amount of lipophilicity and were not dynamic in MES and ScPTZ tests.

\section{CONCLUSION}

In the present investigation, novel 1,2,4triazolo-1,3,4-thiadiazoles was successfully prepared and estimated for anticonvulsant activity by MES and scPTZ tests. Entire compounds displayed moderate to good activity. Preliminary evaluation indicates the target compounds $5 \mathrm{e}, 5 \mathrm{~g}$ and $5 \mathrm{i}$ exhibited potent anticonvulsant activity at a lower dosage $(30 \mathrm{mg} /$ $\mathrm{kg})$. The molecules like $5 \mathrm{a}, 5 \mathrm{~d}, 5 \mathrm{e}, 5 \mathrm{f}, 5 \mathrm{~g}, 5 \mathrm{i}$ and $5 \mathrm{l}$ exhibited activity at 0.5 and $4.0 \mathrm{~h}$ in contrast to seizures it may would-be worth as prototypic candidates. The anticonvulsant data shown that every compound showed distinctive decrease of hind limb tonic extensor stage. Moreover, anticonvulsant activities of the other tested compounds were found to be much less effective than standard drugs (phenytoin and carbamazepine). According to the results obtained it seems that presence of halo-substituted aryl at benzoxazole and hydroxyl and aldehyde substituted aryl at triazolo-thiadiazole moiety displayed the best anticonvulsant activity and favorable high protection. Compounds $5 \mathrm{a}, 5 \mathrm{~b}$, $5 d, 5 e, 5 f, 5 g, 5 i$ and $5 \mathrm{l}$ supposedly were more lipophilic character having strong anticonvulsant activity. Compounds $5 \mathrm{j}$ and $5 \mathrm{k}$ were a lesser amount of lipophilicity and a reduced amount of activities in MES test. Subsequently, triazolo-thiadiazoles were found having anticonvulsant properties, and express to a favorable candidates with fascinating pharmacological values.

\section{ACKNOWLEDGEMENT}

Authors are sincerely thanks to IIT Delhi and Jamia Hamdard University for spectral analysis of the compounds. We extend our sincere thanks to the Antiepileptic Drug Development program for completing the anticonvulsant test.

\section{Conflict of interest}

No conflict of interest exists among authors.

\section{REFERENCES}

1. Njamnshi, A. K.; Bissek, A. C. Z. K.; Yepnjio, F. N.; Tabah, E. N.; Angwafor, S. A.; Kuate, C. T.; Dema, F.; Fonsah, J.Y.; Acho, A.; Kepeden, M. N.; Azinwi, Y. H.; Kuwoh, P. B,; Angwafor, F. F.; Muna, W. F. Epi. Behav., 2010, 17, 95-102.
2. Bell, G. S.; Sander, J. W. Seizure., 2002, 11, 306-314.

3. Husain, A.; Siddiqui, N.; Sarafroz, M.; Khatoon, Y.; Rasid, M.; Ahmad, N. Acta. Pol. Pharma. Drug Res., 2011, 68, 657-663. 
4. Siddiqui, N.; Pandeya, S. N.; Khan, S. A.; Stables, J.; Rana, A.; Alam, M. Bioorg. Med. Chem. Lett., 2007, 17, 255-259.

5. Sabers, A.; Gram, L. Drugs., 2000, 60, 23-33.

6. White, H. S. Epilepsia., 2003, 44, 2-8.

7. Belcastro, V.; Striano, P.; Gorgone, G.; Costa, C.; Ciampa, C.; Caccamo, D.; Pisani, L. R.; Oteri, G.; Marciani, M. G.; Aguglia, U.; Striano, S.; lentile, R.; Calabresi, P.; Pisani, F. Epilepsia., 2010, 51, 274-279.

8. Meador, K. J. J. Clin. Psychi., 2003, 64, 30-34.

9. Leppik, I. E. Epilepsia., 1994, 35, 29-40.

10. Brodie, M. Lancet., 1992, 339, 1397-1400.

11. Szelenyi, I.; Horvath, K.; Howes, J. F.; Mazarat, A. M. Drugs Fut., 2003, 28, 925-936.

12. Li, Y.; Liang, J.; Siu, T.; Hu, E.; Rossi, M. A.; Barnett, S. F.; Defeo-Jones, D.; Jones, R. E.; Robinson, R. G.; Leander, K.; Huber, H. E.; Mittal, S.; Cosford, N.; Prasit, P. Bioorg. Med. Chem. Lett., 2009, 19, 834-836.

13. Kumar, H.; Javed, S. A.; Khan, S. A.; Amir, M. Eur. J. Med. Chem., 2008, 43, 2688-2698.

14. Salgın-Goksen, U.; Gokhan-Kelekci, N.; Goktas, O.; Koysal, Y.; Kilic, E.; Isik, S.; Aktay, G.; Ozalp, M. Bioorg. Med. Chem., 2007, 15, 5738-5751.

15. Gumrukcuoglu, N.; Bekircan, O.; Serdar, M.; Celik, E.; Sevim, A.; Demirbas, N. Turk. J. Chem., 2007, 31, 335-348.

16. Guzeldemirci, N. U.; Kucukbasmaci, O. Eur. J. Med. Chem., 2010, 4, 63-68.

17. Siddiqui, A. A.; Arora, A.; Siddiqui, N.; Misra, A. Ind. J. Chem., 2005, 44, 838-841.

18. Chai, B.; Qian, X.; Cao, S.; Liu, H.; Song, G. Arkivoc., 2003, 2, 141-145.

19. Ma, Y.; Liu, R.; Gong, X.; Li, Z.; Huang, Q.; Wang, H.; Song, G. G. J. Agri. Food. Chem., 2006, 54, 7724-7728.

20. Nagai, S. I.; Ueda, T.; Sugiura, S.; Nagatsu, A.; Murakami, N.; Sakakibara, J.; Fujita, M.; Hotta, Y. J. Het. Chem., 1998, 35, 325-327.

21. El-Shehry, M. F.; Abu-Hashem, A. A.; ElTelbani, E. M. Eur. J. Med. Chem., 2010, 45, 1906-1911.

22. Metwally, K. A.; Yaseen, S. H.; Lashine, E. S. M.; El-Fayomi, H. M.; El-Sadek, M. E. Eur. J. Med. Chem., 2007, 42, 152-160.

23. Amir, M.; Kumar, H.; Javed, S. A. Eur. J. Med. Chem., 2008, 43, 2056-2066.
24. Sahu, J. K.; Ganguly, S.; Kaushik, A. J. Adv. Pharma. Tech. Res., 2014, 5(2), 90-95.

25. Karegoudar, P.; Prasad, D. J.; Ashok, M.; Mahalinga, M.; Poojary, B.; Holla, B. S. Eur. J. Med. Chem., 2008, 43(4), 808-815.

26. Ramaprasad, G. C.; Kalluraya, B.; Kumar, B. S.; Mallya, S. Der Pharma. Chemi., 2012, 4(3), 1026-1032.

27. Sunil D, Isloor AM, Shetty P. Synthesis, characterization and anticancer activity of 1,2,4triazolo[3,4-b]-1,3,4-thiadiazoles on Hep G2 cell lines. Der Pharma Chemi., 2009, 1(2), 19-26.

28. Husain, A.; Naseer, M. A.; Sarafroz, M. Acta Pol. Pharma. Drug Res. 2009, 66, 135-140.

29. Plech, T.; Wujec, M.; Kosikowska, U.; Malm, A.; Kapro, B. Eur. J. Med. Chem., 2012, 47, 580-584.

30. Gopalakrishna, B.; Ranghunandan, N.; Rao, V. J.; Bari, S.; Venkatesham, A.; Sarangapani, M. Ind. Drug., 2005, 5, 369-374.

31. El-Shehry, M. F.; Abu-Hashem, A. A.; ElTelbani, E. M. Eur. J. Med. Chem., 2010, 45, 1906-1911.

32. Li, Y. J.; Liu, L. J.; Jin, K.; Xu, Y. T.; Sun, S. Q. Chin. Chem. Lett., 2010, 21, 293-296.

33. Porter, R. J.; Cereghino, J. J.; Gladding, G. D.; Hessie, B.J.; Kupferberg, H. J.; Scoville, B.;White, B. G. Clev. Clin. Qua., 1984, 51, 293-305.

34. Krall, R. L.; Penry, J. K.; White, B. G.; Kupferberg, H. J.; Swinyard, E. A. Epilepsia., 1978, 19, 409-428.

35. Swinyard, E. A.; Woodhead, J. H.; White, H. S.; Franklin, M. R.; Levy, R. H.; Mattson, R. H.; Melrum, B.; Penry, J. K.; Dreifuss, F. E. Raven-Press., 85-102.

36. Dunham, N, W.; Miya, T. S. J. Ame. Pharma. Asso., 1957, 46, 208-209.

37. Lien, E. J.; Liuo, R. C. H.; Shinoucla, H. G. J. Pharma. Sci., 1979, 68, 463-468.

38. Farrar, V. A.; Ciechanowicz-Rutkowska, M.; Grochowski, J.; Serda, P.; Pilati, T.; Filippini, G.; Hinko, C. N.; El-Assadi, A.; Moore, J. A.; Edafiogho, I. O.; Andrews, C. W.; Cory, M.; Nicholson, J. M.; Scott, K. R. J. Med. Chem., 1993, 36, 3517-3525.

39. Reid, J. R.; Heindel, N. D. J. Heterocy. Chem., 1976, 13, 925-926.

40. Dimmock, J. R.; Vashishtha, S. C.; Stables, J. P. Pharmazie., 2000, 55, 490-494. 